ERRATUM

\title{
Interrogating open issues in cancer medicine with patient-derived
} xenografts

Annette T. Byrne, Denis G. Alférez, Frédéric Amant, Daniela Annibali, Joaquín Arribas, Andrew V. Biankin, Alejandra Bruna, Eva Budinská, Carlos Caldas, David K. Chang, Robert B. Clarke, Hans Clevers,

George Coukos, Virginie Dangles-Marie, S. Gail Eckhardt, Eva Gonzalez-Suarez, Els Hermans,

Manuel Hidalgo, Monika A. Jarzabek, Steven de Jong, Jos Jonkers, Kristel Kemper, Luisa Lanfrancone,

Gunhild Mari Mcelandsmo, Elisabetta Marangoni, Jean-Christophe Marine, Enzo Medico,

Jens Henrik Norum, Héctor G. Palmer, Daniel S. Peeper, Pier Giuseppe Pelicci, Alejandro Piris-Gimenez,

Sergio Roman-Roman, Oscar M. Rueda, Joan Seoane, Violeta Serra, Laura Soucek, Dominique Vanhecke,

Alberto Villanueva, Emilie Vinolo, Andrea Bertotti \& Livio Trusolino

Nature Reviews Cancer 17, 254-268 (2017)

In the online html version of this article, Joan Seoane's affiliations were not correct. He is also a member of the EurOPDX Consortium and is at the Vall d'Hebron Institute of Oncology, 08035 Barcelona, the Universitat Autònoma de Barcelona, 08193 Bellaterra, and the Institució Catalana de Recerca i Estudis Avançats (ICREA), 08010 Barcelona, Spain. This is correct in the print and PDF versions of the article. 